\title{
Robustness of Traveling Waves in Ongoing Activity of Visual Cortex
}

\author{
Ian Nauhaus, ${ }^{1}$ Laura Busse, ${ }^{2}$ Dario L. Ringach, ${ }^{3}$ and Matteo Carandini ${ }^{4}$ \\ ${ }^{1}$ Salk Institute for Biological Studies, La Jolla, California 92037, ${ }^{2}$ Werner Reichardt Centre for Integrative Neuroscience, University of Tübingen, 72076 \\ Tübingen, Germany, ${ }^{3}$ Departments of Neurobiology and Psychology, University of California Los Angeles, Los Angeles, California 90095, and ${ }^{4}$ UCL Institute \\ of Ophthalmology, University College London, London EC1V 9EL, United Kingdom
}

Numerous studies have revealed traveling waves of activity in sensory cortex, both following sensory stimulation and during ongoing activity. We contributed to this body of work by measuring the spike-triggered average of the local field potential (stLFP) at multiple concurrent locations (Nauhaus et al., 2009) in the visual cortex of anesthetized cats and macaques. We found the stLFP to be progressively delayed at increasing distances from the site of the triggering spikes, and interpreted this as a traveling wave of depolarization originating from that site. Our results were criticized, however, on two grounds. First, a study using the same recording techniques in the visual cortex of awake macaques reported an apparent lack of traveling waves, and proposed that traveling waves could arise artifactually from excessive filtering of the field potentials (Ray and Maunsell, 2011). Second, the interpretability of the stLFP was questioned (Kenneth Miller, personal communication), as the stLFP must reflect not only interactions between spike trains and field potentials, but also correlations within and across the spike trains. Here, we show that our data and interpretation are not imperiled by these criticisms. We reanalyzed our field potentials to remove any possible artifact due to filtering and to discount the effects of correlations within and across the triggering spike trains. In both cases, we found that the traveling waves were still present. In fact, closer inspection of Ray and Maunsell's (2011) data from awake cortex shows that they do agree with ours, as they contain clear evidence for traveling waves.

\section{Introduction}

Measurements in multiple cortical areas have revealed patterns of activity organized as traveling waves (Wu et al., 2008; Mohajerani et al., 2010). In visual cortex, in particular, focal visual stimuli elicit activity that spreads over time. This gradual spread has been inferred from local measurements (Kitano et al., 1995; Bringuier et al., 1999) and has been observed directly by imaging voltagesensitive dyes (Grinvald et al., 1994; Jancke et al., 2004; Benucci et al., 2007; Sharon et al., 2007; Sit et al., 2009).

Traveling waves also occur in visual cortex during ongoing activity (Nauhaus et al., 2009). Ongoing activity is believed to reflect the basic organization of the cortical circuitry (Tsodyks et al., 1999; Kenet et al., 2003; Destexhe and Contreras, 2006; Haider and McCormick, 2009; Ringach, 2009). It is thought to be dominated by lateral connections, whereas driven activity in the presence of strong visual stimuli is thought to reflect largely subcortical inputs and local computations (Kitano et al., 1994, 1995; Haider and McCormick, 2009; Nauhaus et al., 2009).

Received Nov. 22, 2011; revised Jan. 12, 2012; accepted Jan. 17, 2012.

Author contributions: I.N., D.L.R., and M.C. designed research; I.N., L.B., D.L.R., and M.C. performed research; I.N. and M.C. analyzed data; I.N., L.B., and M.C. wrote the paper.

This work was supported by the Medical Research Council (G0800791) and by the European Research Council (project CORTEX). I.N. was supported by an National Research Service Award Fellowship (EY019821) and L.B. was supported by funds awarded to the Centre for Integrative Neuroscience (DFG Exec 307). M.C. holds the GlaxoSmithKline Fight for Sight Chair in Visual Neuroscience. We thank Michael Okun for useful suggestions.

Correspondence should be addressed to Matteo Carandini, UCL Institute of Ophthalmology, 11-43 Bath Street, University College London, London EC1V 9EL, United Kingdom. E-mail: matteo@carandinilab.net.

DOI:10.1523/JNEUROSCI.5827-11.2012

Copyright $\odot 2012$ the authors $\quad 0270-6474 / 12 / 323088-07 \$ 15.00 / 0$
Our group has contributed to these views by reporting traveling waves during ongoing activity, whose amplitude and spatial footprint are markedly reduced by visual stimulation (Nauhaus et al., 2009). In that study, we monitored populations of neurons in multiple locations by recording field potentials concurrently in each of these locations. We then averaged each local field potential by aligning it to the spontaneous occurrence of spikes in a single designated location. The resulting spike-triggered average of the local field potential, or stLFP (Okun et al., 2010), was large at the triggering location and progressively smaller and increasingly delayed at more distant locations. Visual stimulation greatly decreased the stLFP, both in amplitude and in spatial extent.

Our results, however, were questioned by a study that attempted to replicate our measurements in awake macaques and reported an apparent discrepancy (Ray and Maunsell, 2011). This study proposed that our findings could arise from a simple artifact: excessive filtering of the field potentials.

Moreover, a general criticism was raised as to the interpretation of stLFP data (Kenneth Miller, personal communication). Triggering on spike trains will reflect not only interactions between spike trains and field potentials, but also correlations in the spike trains themselves, over space and time. The effect of these correlations must be removed before conclusions can be drawn about the interactions between spike trains and field potentials.

Here we show that our data and interpretation are not imperiled by these two criticisms. We reanalyzed our local field potential measurements to prevent the possible artifact raised by Ray and Maunsell (2011) and to discount the effects of correlations 

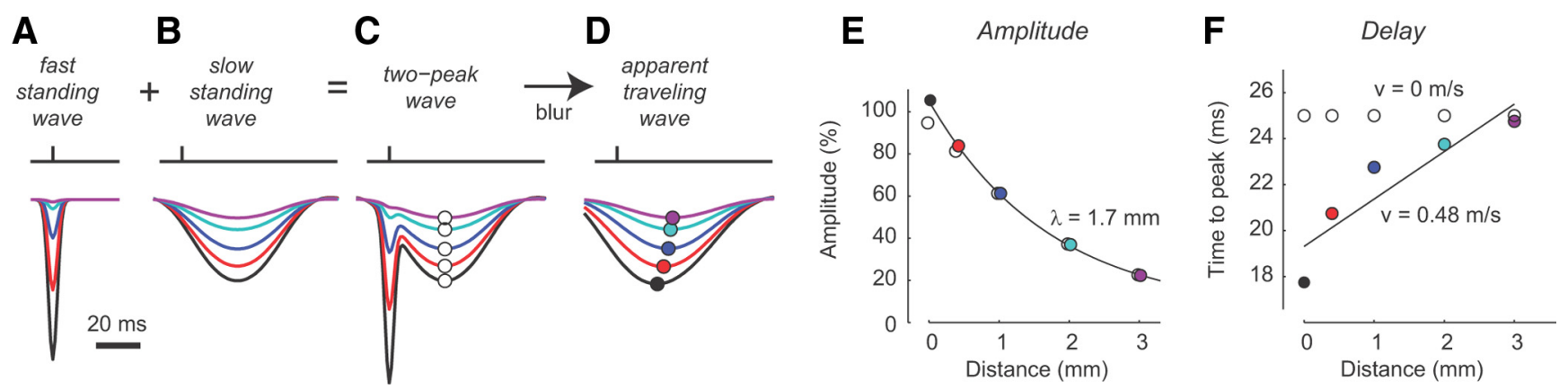

Figure 1. The two-peak hypothesis of Ray and Maunsell (2011). $\boldsymbol{A}$, Fast standing wave. Curves correspond to distances of $0,0.4,1,2$, and $3.0 \mathrm{~mm}$ from the spike-triggering electrode (this color code is maintained throughout all figures). Top trace indicates the time of the triggering spike. $\boldsymbol{B}$, Slow standing wave. $\boldsymbol{C}$, Sum of the two waves. Circles indicate the peaks of the slow component. $\boldsymbol{D}$, Same data, but excessively blurred with cutoff at $20 \mathrm{~Hz}$ to obtain an apparent traveling wave. Circles indicate the peaks of the blurred wave. $\boldsymbol{E}, \boldsymbol{F}$, Dependence of peak amplitude and peak time on distance from the triggering spikes for the slow component of the two-peak wave (open symbols) and for the apparent traveling wave obtained by blurring (filled symbols). The amplitude of the slow wave is fitted with an exponential function with space constant $\lambda$. The delay of the slow wave is fitted with a line with slope $v$ (velocity).

Table 1. Summary of datasets

\begin{tabular}{lrll}
\hline & Spikes & Seconds & $\begin{array}{l}\text { Triggering } \\
\text { neurons }\end{array}$ \\
\hline Monkey A & 57,789 & 300 & 61 \\
Monkey B & 9,201 & 214 & 49 \\
Cat A & 25,526 & 347 & 69 \\
Cat B & 27,860 & 519 & 41 \\
\hline
\end{tabular}

within spike trains pointed out by Miller. In both cases, we found that the traveling waves were still present and that the same conclusions could be drawn as those in our original study (Nauhaus et al., 2009). In fact, we argue that the data of Ray and Maunsell (2011) do agree with ours, and indeed provide evidence for traveling waves of activity in the awake cortex.

\section{Materials and Methods}

We reanalyzed data acquired for our previous study (Nauhaus et al., 2009) and data published by Ray and Maunsell (2011). Briefly, $10 \times 10$ electrode arrays (Blackrock Microsystems) were implanted in the primary visual cortex, acutely in our study (in two female cats and two male macaques), and chronically in Ray and Maunsell (2011) (in two male macaques). For each electrode yielding well sorted spikes, the spike times were used to compute the stLFP across all sites. Recordings were made under anesthesia in our study and during wakefulness in their study. Ongoing activity was measured during the presentation of a gray screen and collected over extended periods of time in our study and during brief $(0.6 \mathrm{~s})$ intervals between successive stimuli in their study.

Test of the two-peak hypothesis. Ray and Maunsell (2011) proposed that traveling waves appear artifactually in the stLFP because of excessive blurring of field potentials (Fig. 1). We therefore repeated our analysis of the stLFP using their suggested high-cut frequency of 500 $\mathrm{Hz}$ (rather than $90 \mathrm{~Hz}$ as in our previous study). The low-cut frequency was at $1 \mathrm{~Hz}$, as lower frequencies are not of interest here. In addition, to make our analysis comparable to that of Ray and Maunsell (2011), we triggered on spikes of well isolated units (rather than multiunit activity) and triggered on all sites where such units were present (rather than selecting sites). As in our original study, we analyzed data from two monkeys and two cats (Table 1).

Spike-field coherence. Similar to Ray and Maunsell (2011), we computed the coherence between spike trains and field potentials. The coherence quantifies the phase consistency between the two signals in different frequency bands and depends on three power spectra: the cross-spectrum and the two auto-spectra. To compute these, we used the multitaper method within overlapping windows in time (Thomson, 1982; Mitra and Pesaran, 1999). Specifically, we applied a $1 \mathrm{~s}$ window, shifted every $0.5 \mathrm{~s}$. Within each $1 \mathrm{~s}$ window, we subtracted the mean and multiplied by the first three Slepian functions ( $2 \mathrm{~Hz}$ bandwidth). We then computed the power spectra by summing the spectra across windows and time shifts.

Traveling waves in the awake cortex. To reanalyze the data published by Ray and Maunsell (2011), we acquired data points from their Figure $2 E$ (with the Matlab function grabit). This figure panel reports stLFP measurements from their Monkey 2, which is the one that delivered most of the data ( 60 triggering electrodes vs 23 for Monkey 1 ) and therefore the most reliable results.

Relation between spike trains and field potentials. The stLFP is a crosscorrelation between spike trains in one place and field potential in another place. Its interpretation is particularly immediate if the relationship between these quantities is linear, i.e., if the field potential $f(x, t)$ measured at a (two-dimensional) location $x$ and time $t$ is a linear transformation of the spike trains $s(x, t)$ occurring at other locations:

$$
f(x, t)=\sum_{d, \tau} k(d, \tau) s(x-d, t-\tau)
$$

Here, $k(d, \tau)$ is the spatiotemporal impulse response (the quantity of interest). The stLFP is an estimate of this impulse response, but this estimate will be correct only if the triggering spike trains are independent white-noise processes or Poisson processes. In the general case, however, estimating the impulse response requires taking into consideration the correlations within and across spike trains.

The impulse response can often be computed by simply taking $K=$ $F / S$, where capital letters indicate Fourier transforms. Here, however, missing electrodes and the spatial boundary of the array create artifacts in the spectral structure of the spike trains and field potentials. These problems can be alleviated by computing the impulse response via the autocorrelation of spike trains and the cross-correlation between the spike trains and field potentials. In particular, the impulse response can also be obtained from $K=F S^{\prime} / S S^{\prime}=C / A$, where prime indicates the complex conjugate and $C$ and $A$ are the Fourier transforms of the cross-correlation and autocorrelation functions, respectively. These more general equations can be estimated without the problem of missing electrodes and with more suitable boundaries.

Unlike our measurements of the raw stLFP, which yielded a separate spatiotemporal kernel for each triggering electrode (Nauhaus et al., 2009), this framework estimates a single spatiotemporal impulse response that is invariant across the array. We computed two of these impulse responses: one during ongoing activity and one during driven activity (obtained by considering all the responses to drifting gratings of various orientations).

Specifically, we defined the cross-correlation and autocorrelation with the following equations:

$$
c(x, t)=\frac{T(x, t)}{N_{c}(x, t)} \sum_{d, \tau} f(d, \tau) s(x+d, t+\tau)
$$




$$
\begin{array}{r}
a(x, t)=\frac{T(x, t)}{N_{a}(x, t)} \sum_{d, \tau} s(d, \tau) s(x+d, t \\
+\tau)
\end{array}
$$

Here, the field potential $f$ was $z$-scored at each electrode, and the mean was removed from the spike train vectors $s$. The normalization functions $N_{\mathrm{c}}(x, t)$ and $N_{\mathrm{a}}(x, t)$ account for the different numbers of overlapping elements that result from different shifts and for electrodes that did not yield well isolated neurons. Finally, the function $T(x, t)$ tapers to zero at long delays and distances (it is a Gaussian with $\mathrm{SD}=1.6$ $\mathrm{mm}$ in space and $1.5 \mathrm{~s}$ in time). Multiplying by it did not significantly alter the shape of the correlation functions, but did reduce the high variability present at large distances or long delays, where correlations are based on a smaller number of samples. This procedure improved the stability in identifying the impulse response in the Fourier domain.

To avoid instability in our estimate of the impulse response, we took an additional precaution. Unlike for the stLFP, we low-pass filtered all traces with a cutoff of $125 \mathrm{~Hz}$ and downsampled to $250 \mathrm{~Hz}$. This procedure did not noticeably alter the power spectra, but it prevented instability at high frequency, where both the numerator and the denominator have little power. The impulse response was later (spline) interpolated to $1 \mathrm{kHz}$ to obtain more precise estimates of delay.

\section{Results}

We start by describing the two-peak hypothesis proposed by Ray and Maunsell (2011) as an artifactual source of traveling waves. Next, we test it on our own data from anesthetized animals, and disprove it. Then, we test it on their own data from awake animals, and also disprove it. Finally, we evaluate the criticism by Miller, and find that though it is a reasonable concern, controlling for it leaves the conclusions of our study unaltered.

\section{Test of the two-peak hypothesis}

Ray and Maunsell (2011) proposed a twopeak hypothesis (Fig. 1) to explain the stLFP data that we obtained in anesthetized cats and monkeys (Nauhaus et al., 2009). Under this hypothesis, the stLFP is not a traveling wave but rather the sum of two standing waves: a fast standing wave that coincides with the spike (Fig. $1 A$ ) and a slow standing wave that follows it by a fixed delay (Fig. $1 B$ ). Both standing waves are strongest at the triggering electrode, and progressively weaker with increasing distance, but the fast wave decreases more rapidly with distance. Their sum is a two-peak waveform, in which the timing of neither peak shows any variation with cortical distance (Fig. 1C). However, blurring this wave by excessive lowpass filtering would merge the two peaks and create an artifactual traveling wave (Fig. $1 D$ ). While the true peak of the slow wave has the same delay at all cortical distances (Fig. $1 F$,
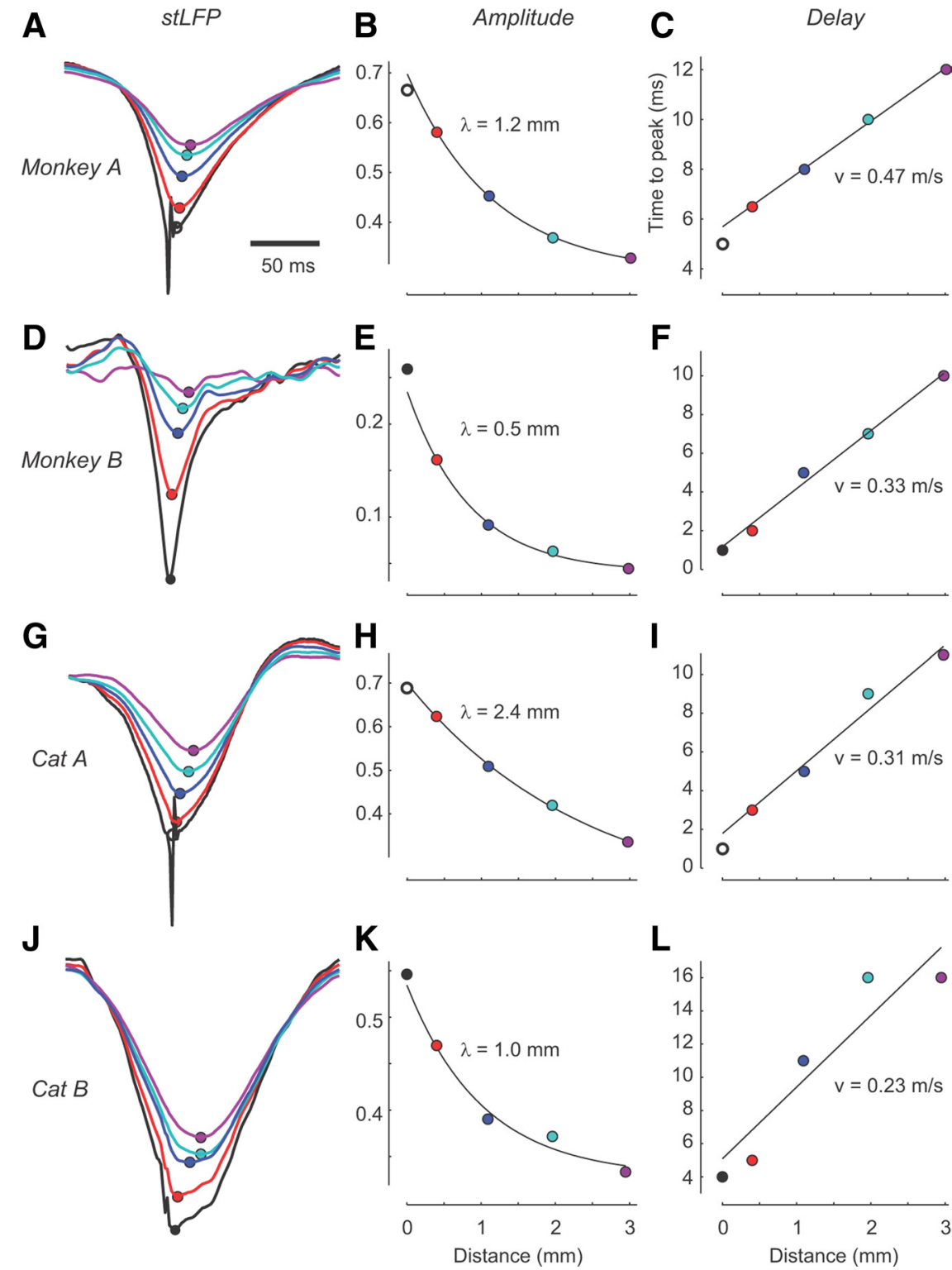

Figure 2. Traveling waves in anesthetized cortex. $\boldsymbol{A}$, stLFP measured at various distances from the triggering electrode in an anesthetized monkey. Field potentials were obtained by filtering the recordings with a high-cut frequency of $500 \mathrm{~Hz}$ to test the two-peak hypothesis. Traces are averages of the stLFP triggered by all electrodes that had well isolated single units (Table 1). Original data are from our previous study (Nauhaus et al., 2009). Dots were added to indicate the minima of the slow wave. $\boldsymbol{B}$, Amplitude of the slow wave as a function of distance from triggering electrode. Curve is best fitting exponential function. The space constant is indicated. $C$, Delay of the slow wave as a function of distance from triggering electrode. Line is the best linear fit. Its slope is $1 / v$, where $v$ is the speed of travel. $\boldsymbol{D}-\boldsymbol{L}$, Same for the three other animals (one monkey and two cats). White dots (monkey $A$ and cat $A$ ) indicate events where the peaks of the slow and fast waves overlap at the triggering electrode. These values are noisier so we ignored them when fitting the exponential and linear functions to amplitude and delay.

open symbols), the peak of the blurred wave depends on distance (Fig. $1 F$, closed symbols).

The premise of this hypothesis is debatable, because it requires a large amount of blurring. Indeed, to make the simulation (Fig. $1 D, F$ ), we had to use a cutoff frequency as low as $20 \mathrm{~Hz}$ (much lower than the $90 \mathrm{~Hz}$ used in our previous study). Without this pronounced blurring, the model would predict delays of only a couple of milliseconds (corresponding to very fast speeds of apparent propagation).

More importantly, the two-peak hypothesis is not supported by our data (Fig. $2 A-C$ ). To test it, we reanalyzed our data by filtering the local field potentials at $500 \mathrm{~Hz}$ rather than $90 \mathrm{~Hz}$. As 
expected, a frequency of $500 \mathrm{~Hz}$ is so high that it does not remove the triggering spike itself (Zanos et al., 2011); indeed, a fast deflection at the time of the spike appears for the triggering site (Fig. $2 A)$. This fast deflection, however, is present only in the triggering electrode. The traces of all other electrodes have only a single peak, the peak of a slow wave. As distance from the triggering electrode increases, this peak gets progressively smaller (Fig. 2B) and delayed (Fig. 2C), indicating a traveling wave. For comparison, under the two-peak hypothesis (Fig. 1C), the slow wave should have been a standing wave, i.e., one whose time of peak would not vary with distance (Fig. $1 F$ ).

Similar results were obtained in the remaining three animals (Fig. 2D-L). Increasing the cutoff filter to $500 \mathrm{~Hz}$ revealed a sharp deflection in all but one animal (monkey B), and only at the triggering location, where it was most likely a reflection of the spike itself. Once again, all the remaining traces had a single peak, the height of this peak decreased with distance, and the delay of the peak increased with distance. These observations contradict the two-peak hypothesis and support the view of traveling waves propagating from the site of the triggering spikes (Nauhaus et al., 2009).

In fact, these data also allow us to examine an additional notion put forward by Ray and Maunsell (2011): that the stLFP is dominated by network rhythms, i.e., global phase-locking between spikes and LFP oscillations. A simple examination of our unfiltered stLFP waveforms well before and after their peak (Fig. 3 , left) reveals that they constitute a unimodal deflection, not an oscillation as would have been expected if they exhibited rhythmic activity. Indeed, the coherence between spike and field exhibited is largest at the lowest frequencies, revealing no notable peaks at intermediate frequencies (Fig. 3, right). This constitutes evidence against oscillations.

\section{Traveling waves in the awake cortex}

Having found that the two-peak hypothesis does not apply to our data in anesthetized animals, we asked whether it is supported by the data obtained in awake animals by Ray and Maunsell (2011). If so, this discrepancy could point to a fundamental difference between the awake cortex and the anesthetized cortex.

We considered the data published by Ray and Maunsell (2011), and specifically the average time courses of the stLFP measured at various distances from the triggering electrode (Fig. $4 A$ ). These data resemble those that we had measured in anesthetized animals (Fig. 2), with some minor differences. First, the delay between the sharp transient and the slow wave at the triggering electrode was $\sim 20 \mathrm{~ms}$ in the awake monkey (Fig. $4 \mathrm{~A}$ ), longer than the 1-5 ms measured between spike and slow wave in anesthetized animals. Second, the sharp transient was present in the awake animal not only at the triggering site, where it probably reflects the triggering spike (Zanos et al., 2011), but also in the immediately neighboring electrodes, where it may reflect synchronous spikes (Singer, 1999).

Though at first glance these awake data may seem to support the two-peak hypothesis, a closer inspection reveals otherwise (Fig. 4). There do appear to be two waves, one fast and one slow, but only the fast wave is synchronous across space (a standing wave). The time course of the slow wave, instead, clearly depends on space (Fig. 4A; replotted from Ray and Maunsell, 2011). This dependence resembles the one seen in our measurements from anesthetized animals (Fig. 2) and is inconsistent with the key aspect of the two-peak hypothesis (Fig. 1). Indeed, in the awake animal, increasing the distance from the triggering electrode caused the peak of the slow wave to decrease exponen-
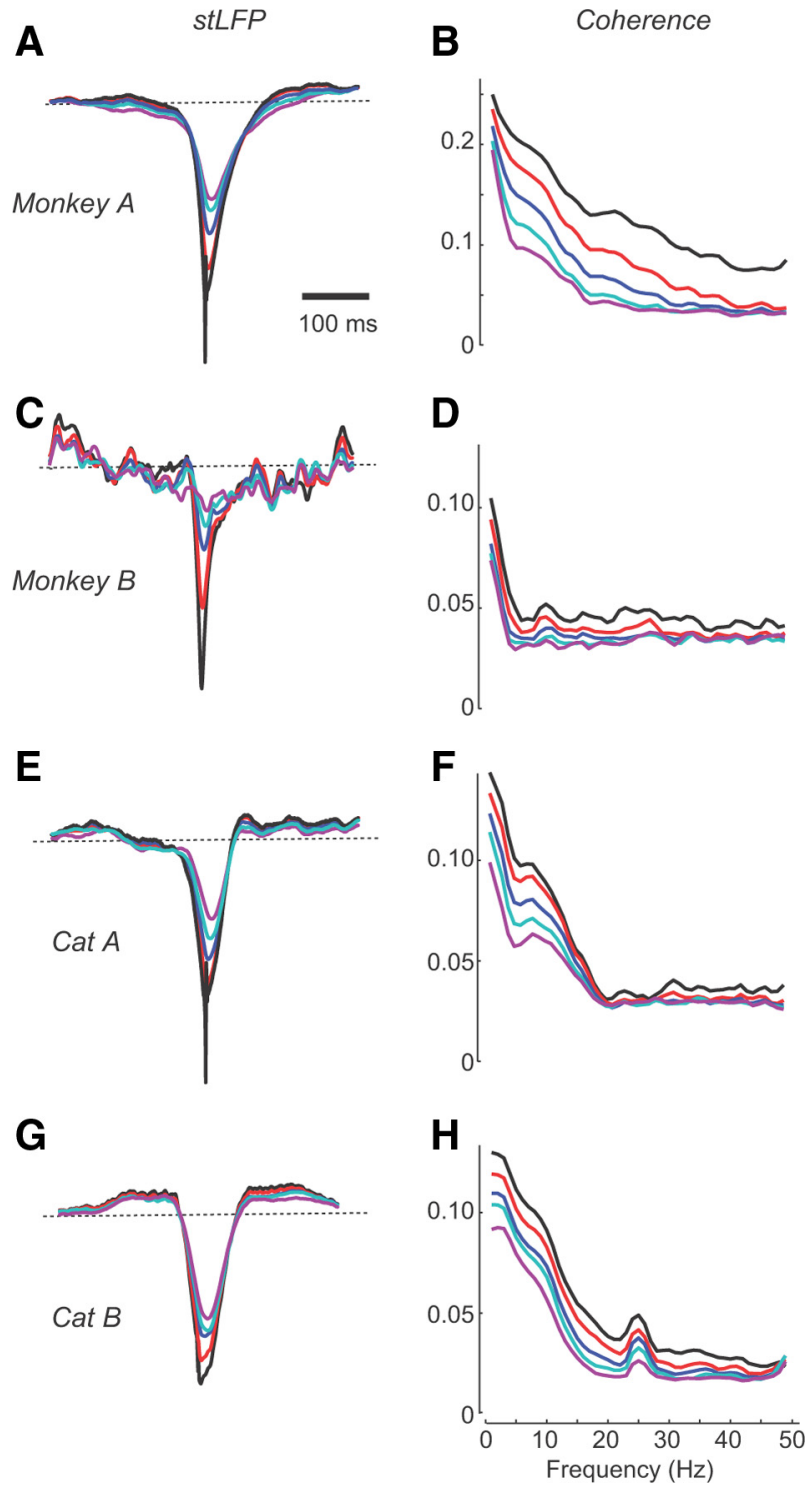

Figure 3. Lack of network rhythms. $\boldsymbol{A}, \boldsymbol{C}, \boldsymbol{E}, \boldsymbol{G}$, The same waveforms as Figure 2, shown over a longer time window ( $\pm 250 \mathrm{~ms}$ ). Dashed lines indicate zero on the ordinate. $\boldsymbol{B}, \boldsymbol{D}, \boldsymbol{F}, \boldsymbol{H}$, Spike-field coherence as a function of frequency (abscissa) and distance (colors).

tially in amplitude (Fig. $4 B$ ) and to increase progressively in delay (Fig. 4C).

So, once again, the stLFP is a traveling wave. In other words, the data of Ray and Maunsell (2011) from awake monkey, far from supporting the two-peak hypothesis, provide evidence for traveling waves similar to those we had observed in anesthetized cats and monkeys.

\section{Relation between spike trains and field potentials}

The interpretation of the stLFP is particularly ready if there is a linear transformation between spike trains at one location and field potential at another location. This transformation is characterized by an impulse response describing the impact of a spike on the field potential (as a function of distance and delay from the spike). The impulse response summarizes the coupling between spike trains and field potentials across space and time.

The stLFP constitutes an approximation of this impulse response, but this approximation can be accurate only if the spike trains are uncorrelated across space and time. In reality 
this assumption is unlikely to hold: spikes can be correlated in space and in time (Cohen and Kohn, 2011). Therefore, as remarked by Kenneth Miller (personal communication), any spatiotemporal structure found in the stLFP may reflect structure in the spike trains rather than in the relationship between spike trains and field potential.

To obtain a closer approximation of the impulse response relating spikes to field potentials, we corrected for the autocorrelation of the spike trains computed over space and time (Fig. 5). This analysis gives us a single point-spread function for each of our experiments. We performed it for Monkey A (Fig. 5A-E) and Cat A (Fig. 5F-J), as these had the largest number of triggering neurons (Table 1) and thus yielded the most robust estimates of the autocorrelation function of the spike trains.

Reassuringly, the salient aspects of the impulse response (Fig. 5) resemble those that we had observed in the raw stLFP (Fig. 2). The autocorrelation of the spike trains appeared to be mostly a delta function surrounded by small and broad flanks (Fig. 5A,E), suggesting that it would not introduce much distortions in the stLFP. Indeed, the impulse response resembled the raw stLFP (Fig. 2): it was a slow wave that was progressively smaller indicates the speed of travel. and more delayed with increasing distance from the triggering spikes (Fig. $5 B, G$ ). The amplitude decayed exponentially with distance (Fig. $5 D, I$ ), and the delay increased fairly linearly (Fig. $5 E, J)$, indicating a speed of $0.29 \mathrm{~m} / \mathrm{s}$ for the monkey and $0.18 \mathrm{~m} / \mathrm{s}$ for the cat. As with the raw stLFP, therefore, the impulse response constitutes a traveling wave.

The impulse response is particularly useful to study how the relation between spikes and field potential may depend on visual

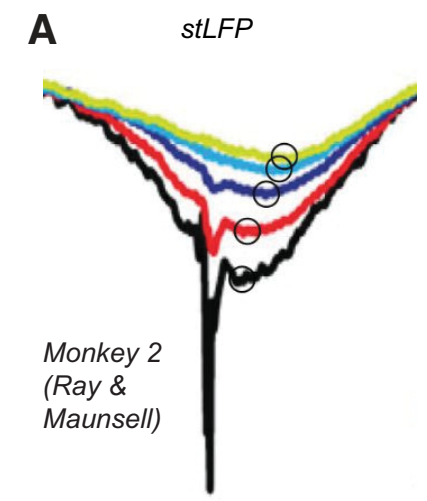

B Amplitude

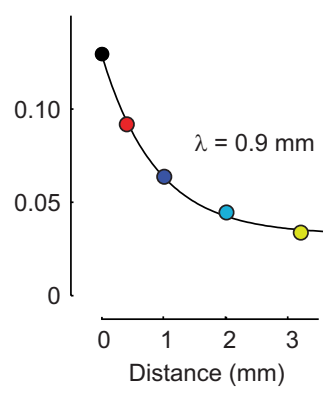

C Delay

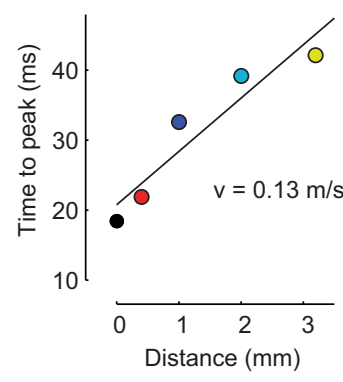

Figure 4. Presence of traveling waves in awake cortex. A, Average of spike-triggered LFP measured at various distances from the triggering electrode, measured in awake monkeys by Ray and Maunsell (2011). Data are repotted from their Figure $2 E$. Dots are added to indicate the minima of the slow wave. $\boldsymbol{B}$, Amplitude of the slow wave as a function of distance. Curve indicates the fit of an exponential function. $\boldsymbol{C}$, Dependence of delay on distance from triggering electrode. The line is the best linear fit. Its slope

Spike autocorrelation

A

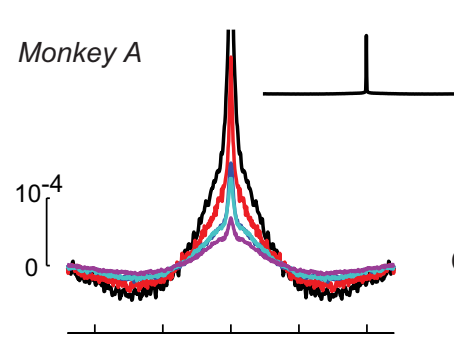

B Ongoing

Impulse response
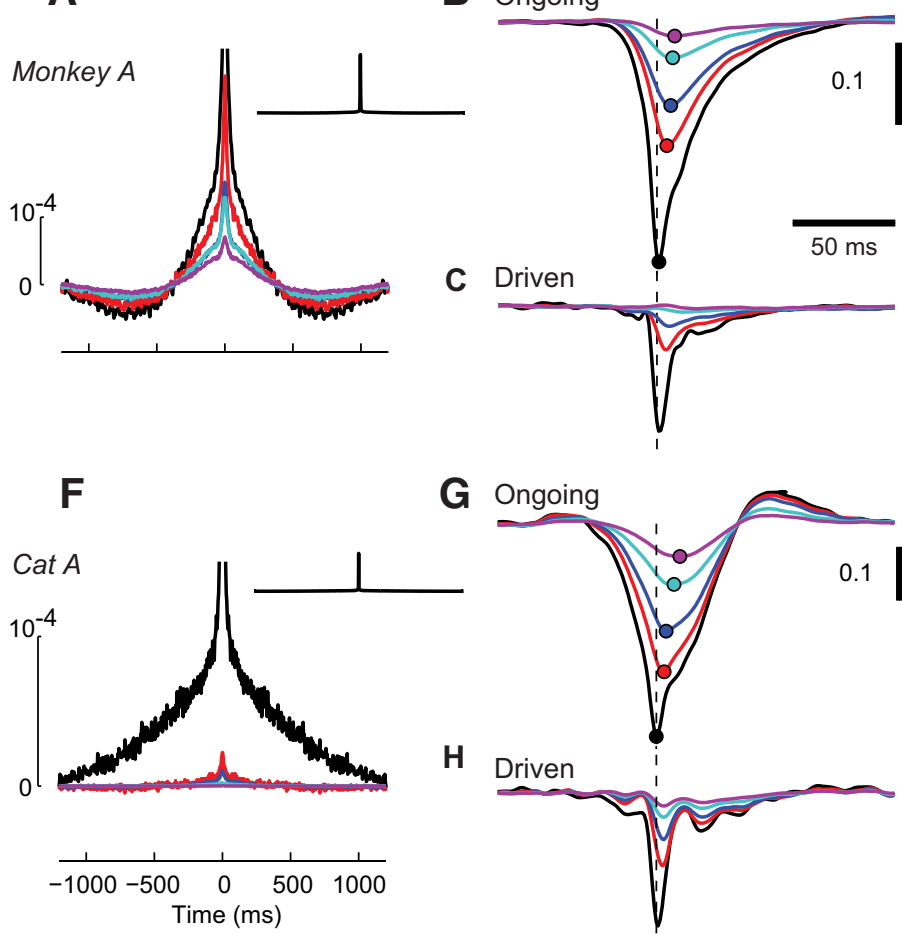

Amplitude

D

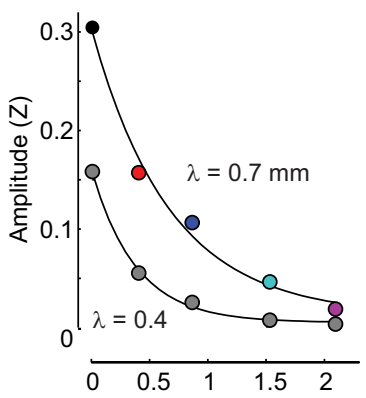

I

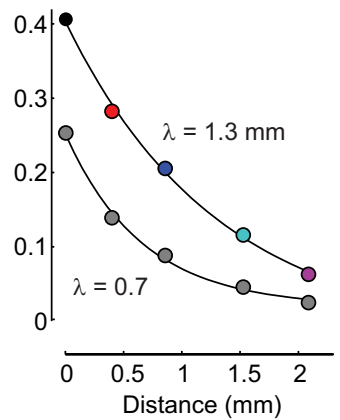

Delay

\section{E}

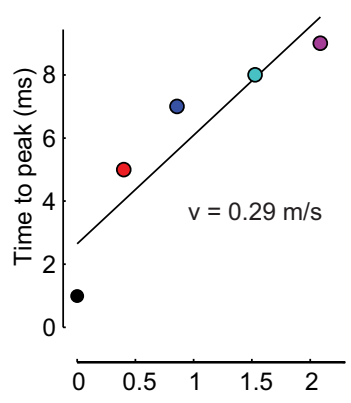

J

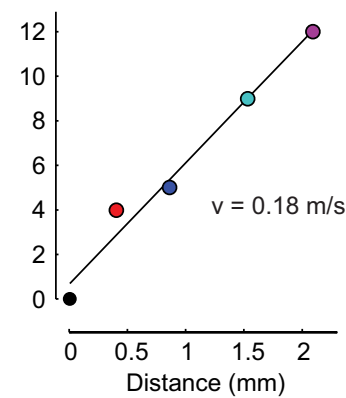

Figure 5. The impulse response of the transformation between spike trains and field potentials. $A$, Autocorrelation of the spike trains for monkey $A$, as a function of time (abscissa) and distance (colors, as in Fig. 1). The units are given by the autocorrelation equation (see Materials and Methods). The peak at $d=0$ was clipped to show the shape of the waveform. Insets show the full shape, which resembles a delta function. $\boldsymbol{B}$, Impulse responses for monkey $A$ estimated during ongoing activity, at various distances from the triggering electrode. This impulse response resembles the raw stLFP (Fig. 2A). The dashed line indicates the spike time. $\boldsymbol{C}$, Same as $\boldsymbol{B}$, for driven activity, i.e., during stimulation with full contrast, large gratings (Nauhaus et al., 2009). $\boldsymbol{D}$, Dependence of amplitude on distance for ongoing activity (colored points), and for driven activity (gray points). Exponential decay functions fit the data (space constant as indicated). $\boldsymbol{E}$, Same as $\boldsymbol{D}$, for time-to-peak. $\boldsymbol{F}$-J, as in $\boldsymbol{A}-\boldsymbol{E}$, respectively, for Cat $A$. 
stimulation. In our previous work, we reported that the raw stLFP was substantially modified by visual stimulation: it became smaller and decayed much faster with distance than during ongoing activity (Nauhaus et al., 2009). Since the raw stLFP is blurred by the spike autocorrelation, this effect might be more parsimoniously explained by changes in spike autocorrelation rather than changes in the relationship between spikes and field potentials. This is a reasonable possibility, since spikespike correlations can indeed become weaker when contrast is increased (Kohn and Smith, 2005). The impulse response allows us to distinguish between these two possibilities because, unlike the stLFP, it discounts the effects of the spike autocorrelation.

We therefore analyzed the impulse response during driven activity (Fig. $5 \mathrm{C}, \mathrm{H}$ ) and found that the results mirror those we had reported using the stLFP (Nauhaus et al., 2009). Visual stimulation reduced the amplitude of the impulse responses (Fig. $5 C, H$ ) relative to those measured during ongoing activity (Fig. $5 B, G)$. It also substantially reduced its space constant, from 0.67 to $0.38 \mathrm{~mm}$ in the monkey (Fig. $5 D$ ) and from 1.32 to $0.66 \mathrm{~mm}$ in the cat (Fig. 5I). These results therefore echo those that we had observed in the stLFP (Nauhaus et al., 2009): at the time of spikes there are traveling waves in the field potential, and these traveling waves are reduced in amplitude and dampened in extent in the presence of visual stimuli.

\section{Discussion}

We embarked on this study because our earlier results (Nauhaus et al., 2009) had been challenged by criticisms and by apparently contradictory new data. We found that these criticisms do not affect our original findings, and that the new data, far from being contradictory, are in agreement with ours.

The first criticism that we addressed is Ray and Maunsell's (2011) two-peak hypothesis, in which travel of stLFP arises artifactually from overfiltering two standing waves with different spatial and temporal footprints. We refuted this hypothesis in three ways. First, we performed a simple simulation and found that the two-peak hypothesis could lead to apparent speeds matching our observations only if one blurred the standing waves with an unrealistically low cutoff frequency of $20 \mathrm{~Hz}$ (Fig. 1). Second, we reanalyzed our data with cutoff frequencies as high as $500 \mathrm{~Hz}$, and instead of finding evidence for standing waves we confirmed the presence of robust traveling waves (Fig. 2). Finally, we inspected Ray and Maunsell's (2011) data from awake monkeys and saw that their slow wave is in fact a traveling wave (Fig. 4). These analyses, therefore, not only disprove the two-peak hypothesis but also provide new evidence for traveling waves in awake cortex similar to those we had observed in anesthetized cats and monkeys.

There are minor discrepancies between our stLFP waveforms and those measured by Ray and Maunsell (2011). The main one is that their stLFP waveforms include fast transients not only at the triggering electrode, but also at nearby electrodes. These transients may be spikes of nearby neurons firing in synchrony (Singer, 1999). Fast local synchrony can be particularly marked in awake animals (Destexhe et al., 1999), so perhaps the reason for the discrepancy is the different state of alertness (anesthetized in our study, awake in theirs). However, these discrepancies may also arise from a fundamental difference in experimental conditions: we recorded ongoing activity during blank intervals lasting tens of seconds, whereas Ray and Maunsell (2011) extracted ongoing activity from brief ( $0.6 \mathrm{~s}$ ) blank intervals between flashing stimuli. Such intervals are unusually short for a study of spontaneous activity.

Overall, we found no evidence to support Ray and Maunsell's (2011) notion that the stLFP is dominated by network rhythms. In our data (Fig. 3), the stLFP constitutes a unimodal deflection with little if any trace of oscillations, and the coherence between spike and field does not have any peaks (being simply monotonically decreasing with frequency). These findings may seem to disagree with the data of Ray and Maunsell (2011), but they need not: in those data, the apparent oscillations in stLFP are most likely simply due to high-pass filtering an otherwise unimodal trace. Indeed, before high-pass filtering, the stLFPs measured by Ray and Maunsell (2011) show little evidence for oscillations (their Fig. $2 A, E$ ). Oscillations become prominent only after highpass filtering above $3 \mathrm{~Hz}$ (their Fig. 2C,G). This view is reinforced by the absence of any meaningful peaks in the coherence spectrum between spikes and field potential in their study (Fig. $3 B, E$ ). This coherence is simply highest at the lowest available frequency, which is in turn constrained by the brief duration of the window of analysis. In summary, rather than traveling waves in our study being a result of low-pass filtering, it appears that network rhythms in that study may be a result of high-pass filtering.

There is also no support for Ray and Maunsell's (2011) criticism that our earlier criteria for electrode selection would bias the results toward finding traveling waves. In our study (Nauhaus et al., 2009), we selected triggering electrodes showing a significant correlation between the time-to-peak of the stLFP and distance, to obtain more robust estimates of speed. We did not select based on speed: if there were no traveling waves we would have found average speeds of zero. In any event, the matter is now put to rest, as in the present analyses we used all electrodes as triggers, and we still found strong traveling waves (Fig. 2).

Similarly, we do not find evidence for Ray and Maunsell's (2011) suggestion that our original estimates of the rate of spatial decay of the stLFP for ongoing activity were inflated. Ray and Maunsell (2011) suggest that we overestimated the space constant by fixing the baseline asymptote to that computed for driven activity. The matter is once again put to rest, as in the present analysis we do not follow this procedure and we still confirm our earlier conclusions (Nauhaus et al., 2009). Rather, the discrepancy between our estimates of spatial decay and theirs probably arises from Ray and Maunsell's (2011) treatment of the sharp transients in the stLFP. Given that the quantity of interest is the slow waves (Fig. 1), one should not let the fast transient corrupt estimates of the exponential decay and the speed of travel (Figs. 2, 3). Ray and Maunsell (2011) instead chose minima of the curve regardless of whether they belong to the sharp transient or to the slow wave. As can be readily pictured, this leads to an apparent fast decay of amplitude with space: one is mixing the short space constant of the sharp transient with the longer space constant of the slower, traveling wave. This explains why the estimate of space constant reported by Ray and Maunsell (2011) for Monkey 2 (Fig. $4 B)$ is so much shorter than the one we measured in the same data $(0.3$ vs $0.9 \mathrm{~mm})$.

We have addressed Kenneth Miller's criticism (personal communication) regarding the limitations of stLFP by developing an unbiased approach for measuring spike-triggered quantities. This method reveals an impulse response that is not corrupted by the autocorrelation of the spike trains in space and time. In our data, this impulse response (Fig. 5) had similar properties to the raw stLFP (Fig. 2): it was largest at the location of the triggering 
spike, decayed progressively with distance, and increased in latency with increasing distance. In other words, as we reported in our previous study (Nauhaus et al., 2009), the average field potential that follows a spike is a traveling wave.

While the interpretation of stLFP in terms of intracellular signals is becoming clearer (Okun et al., 2010), a key question remains open: to what degree does the stLFP reveal a causal relationship between spikes and field potential? Our previous study may have overstated the case for causality (Nauhaus et al., 2009). Causality is a reasonable proposal as spikes are expected to cause depolarizations in progressively more distant neurons. For instance, focal visual stimulation elicits outward propagating waves well beyond the classical receptive field (Kitano et al., 1995; Bringuier et al., 1999; Benucci et al., 2007). However, because depolarizations facilitate the making of more spikes, a traveling wave of depolarization is likely to modulate spikes in its wake. One would therefore not expect the observed correlations between spikes and field potentials to be entirely causal. Nonetheless, the mass of impulse response lies overwhelmingly after the time of the spike (92\% in Monkey A and $85 \%$ in cat A; taken for distances $>0 \mathrm{~mm}$ and within a $240 \mathrm{~ms}$ window). The fact that the impulse response appears to be a mostly causal linear filter is consistent with, though it does not establish, such a causal relationship.

Clearly, to address this issue of causality, and more generally to test the degree to which functional connectivity corresponds to causal anatomical connectivity, one will need to intervene in the system and cause the spikes artificially. This could be done through direct focal manipulation of spikes; for example. via electrical stimulation or optogenetics. Until then, one can only speculate on the relationship between spikes and distal fields, and cannot assert with certainty that it is causal and mediated by lateral circuitry.

\section{References}

Benucci A, Frazor RA, Carandini M (2007) Standing waves and traveling waves distinguish two circuits in visual cortex. Neuron 55:103-117.

Bringuier V, Chavane F, Glaeser L, Frégnac Y (1999) Horizontal propagation of visual activity in the synaptic integration field of area 17 neurons. Science 283:695-699.

Cohen MR, Kohn A (2011) Measuring and interpreting neuronal correlations. Nat Neurosci 14:811-819.

Destexhe A, Contreras D (2006) Neuronal computations with stochastic network states. Science 314:85-90.

Destexhe A, Contreras D, Steriade M (1999) Spatiotemporal analysis of local field potentials and unit discharges in cat cerebral cortex during natural wake and sleep states. J Neurosci 19:4595-4608.

Grinvald A, Lieke EE, Frostig RD, Hildesheim R (1994) Cortical point- spread function and long-range lateral interactions revealed by real-time optical imaging of macaque monkey primary visual cortex. J Neurosci 14:2545-2568.

Haider B, McCormick DA (2009) Rapid neocortical dynamics: cellular and network mechanisms. Neuron 62:171-189.

Jancke D, Chavane F, Naaman S, Grinvald A (2004) Imaging cortical correlates of illusion in early visual cortex. Nature 428:423-426.

Kenet T, Bibitchkov D, Tsodyks M, Grinvald A, Arieli A (2003) Spontaneously emerging cortical representations of visual attributes. Nature 425:954-956.

Kitano M, Niiyama K, Kasamatsu T, Sutter EE, Norcia AM (1994) Retinotopic and nonretinotopic field potentials in cat visual cortex. Vis Neurosci 11:953-977.

Kitano M, Kasamatsu T, Norcia AM, Sutter EE (1995) Spatially distributed responses induced by contrast reversal in cat visual cortex. Exp Brain Res 104:297-309.

Kohn A, Smith MA (2005) Stimulus dependence of neuronal correlation in primary visual cortex of the macaque. J Neurosci 25:3661-3673.

Mitra PP, Pesaran B (1999) Analysis of dynamic brain imaging data. Biophys J 76:691-708.

Mohajerani MH, McVea DA, Fingas M, Murphy TH (2010) Mirrored bilateral slow-wave cortical activity within local circuits revealed by fast bihemispheric voltage-sensitive dye imaging in anesthetized and awake mice. J Neurosci 30:3745-3751.

Nauhaus I, Busse L, Carandini M, Ringach DL (2009) Stimulus contrast modulates functional connectivity in visual cortex. Nat Neurosci 12:70-76.

Okun M, Naim A, Lampl I (2010) The subthreshold relation between cortical local field potential and neuronal firing unveiled by intracellular recordings in awake rats. J Neurosci 30:4440-4448.

Ray S, Maunsell JH (2011) Network rhythms influence the relationship between spike-triggered local field potential and functional connectivity. J Neurosci 31:12674-12682.

Ringach DL (2009) Spontaneous and driven cortical activity: implications for computation. Curr Opin Neurobiol 19:439-444.

Sharon D, Jancke D, Chavane F, Na'aman S, Grinvald A (2007) Cortical response field dynamics in cat visual cortex. Cereb Cortex 17:2866-2877.

Singer W (1999) Neuronal synchrony: a versatile code for the definition of relations? Neuron 24:49-65, 111-125.

Sit YF, Chen Y, Geisler WS, Miikkulainen R, Seidemann E (2009) Complex dynamics of $\mathrm{V} 1$ population responses explained by a simple gain-control model. Neuron 64:943-956.

Thomson DJ (1982) Spectrum estimation and harmonic-analysis. Proc IEEE 70:1055-1096.

Tsodyks M, Kenet T, Grinvald A, Arieli A (1999) Linking spontaneous activity of single cortical neurons and the underlying functional architecture. Science 286:1943-1946.

Wu JY, Xiaoying H, Chuan Z (2008) Propagating waves of activity in the neocortex: what they are, what they do. Neuroscientist 14:487-502.

Zanos TP, Mineault PJ, Pack CC (2011) Removal of spurious correlations between spikes and local field potentials. J Neurophysiol 105:474-486. 